\title{
Regulation of gene expression in Mycoplasmas: Contribution from Mycoplasma hyopneumoniae and Mycoplasma synoviae genome sequences
}

\author{
Humberto Maciel França Madeira and Jane Eyre Gabriel \\ Laboratório de Biologia Molecular Aplicada à Agropecuária, Centro de Ciências Agrárias e Ambientais, \\ Pontifícia Universidade Católica do Paraná, São José dos Pinhais, PR, Brazil.
}

\begin{abstract}
This report describes the transcription apparatus of Mycoplasma hyopneumoniae (strains $\mathrm{J}$ and 7448 ) and Mycoplasma synoviae, using a comparative genomics approach to summarize the main features related to transcription and control of gene expression in mycoplasmas. Most of the transcription-related genes present in the three strains are well conserved among mycoplasmas. Some unique aspects of transcription in mycoplasmas and the scarcity of regulatory proteins in mycoplasma genomes are discussed.
\end{abstract}

Key words: Mycoplasma hyopneumoniae, Mycoplasma synoviae, transcription, comparative genomics.

Received: April 12, 2006; Accepted: October 5, 2006.

The recent effort to characterize the minimal gene set required for a living cell on Earth focused on the Mycoplasma genitalium genome data, and compared them to that of another parasitic bacteria, Haemophilus influenzae. The primary assumption was that those bacteria had shed a number of genes in the process of adaptation to the parasitic lifestyle, without losing their fitness (Koonin, 2000). The complete sequencing of the first mollicute in 1995 surprisingly revealed that Mycoplasma genitalium devoted 10 times less genes to regulatory functions than Haemophilus influenzae (5 vs. 64 genes, respectively), despite possessing a genome only three times smaller and having a somewhat similar lifestyle (Fraser et al., 1995). This example highlights the contribution of comparative genomics to the understanding of cell function. The comparative approach allows researchers to predict protein functions by transferring information from functionally characterized proteins of model organisms to their uncharacterized homologs and to delineate the functionally critical parts of protein (and RNA) molecules, such as catalytic or binding sites. In that context, comparative genome research has provided new insights into the accurate mechanisms involved in bacterial gene regulation. Genetic data obtained by genome sequencing of several Mycoplasma strains in the past decade (Fraser et al., 1995; Himmelreich

Send correspondence to Prof Humberto M.F. Madeira. Laboratório de Biologia Molecular Aplicada à Agropecuária, Centro de Ciências Agrárias e Ambientais, Pontifícia Universidade Católica do Paraná, Rodovia BR 376, km 14, 83010-500 São José dos Pinhais, PR, Brazil. E-mail: h.madeira@pucpr.br. et al., 1996; Glass et al., 2000; Chambaud et al., 2001; Sasaki et al., 2002; Papazisi et al., 2003; Jaffe et al., 2004; Minion et al., 2004; Westberg et al., 2004; Vasconcelos et al., 2005) opened an opportunity to explore the functional content of genomes and evolutionary relationships between them at a new qualitative level. Based on recently published data (Vasconcelos et al., 2005), the present report describes the set of genes related to transcription in Mycoplasma hyopneumoniae and Mycoplasma synoviae strains, in a comparative context with other mollicutes. Using standard bioinformatics tools, we aimed at a better understanding of how these bacteria regulate gene expression, and ultimately, how such mechanisms allow them to thrive in their habitats.

Genes involved in the basic cellular processes of transcription and translation comprise about $20 \%$ of the total ORFs in mycoplasmas, similar to results observed in other bacteria (Muto and Ushida, 2002). M. hyopneumoniae strains $\mathrm{J}$ and 7448 and M. synoviae all have $16 \%$ of their genes involved in translation, and the percentage of genes involved in transcription in mycoplasmas ranges from $2-3 \%$, based on COG tables available (National Center of Biotechnology Information). Thus, despite the overall conservation of the percentage of genes involved in translation, a process considered the most highly conserved in eubacteria, the absolute number of genes involved in transcription in mycoplasmas is very low, ranging from 11 to 23. Due to the essential biological role of the transcriptional process, evolutionary deletion of genes in this category had 
to be limited and selective. Based on the COG database (National Center of Biotechnology Information), the number of genes involved in transcription in other bacteria is usually much higher, such as in Neisseria gonorrhoeae (84 genes; $4.2 \%$ of total genes), Haemophilus influenzae (91; $5.3 \%)$, E. coli $(342 ; 8.1 \%)$ and Bacillus subtilis $(372 ; 9 \%)$. Data reveal that the ORFs related to transcription in $M$. hyopnemoniae strains J and 7448 and in M. synoviae code for the bare-bones proteins involved in transcription in prokaryotes (RNA polymerase holoenzyme, including a single $\sigma$ factor, elongation and terminator factors), reinforcing the selectiveness of gene saving in mycoplasmas (Table 1). The number of putative transcriptional regulators in these bacteria is minimal, and this drastic saving in genes raises the questions of how they adapt to environmental changes and how they maintain cellular homeostasis.

The core RNA polymerase of mycoplasmas resembles that of other eubacteria in subunit structure, encoded by the conserved genes rpoA ( $\alpha$ subunit), rроB ( $\beta$ subunit) and rpoC ( $\beta$ ' subunit), as listed in Table 1. The small, accessory $\omega$ subunit (rpoZ), present in many eubacteria, is absent in mycoplasmas. During gene regulation, the sigma factor $(\sigma)$ plays an essential role in the conversion of core
RNA polymerase to holoenzyme, since it directs RNA polymerase to specific promoters, so that transcription initiates at the proper place. As shown in Table 1, the genomes of $M$ hyopneumoniae and $M$. synoviae encode only a single sigma factor, compared to at least 6 in E. coli (Blattner et al., 1997) and 18 in Bacillus subtilis (Kunst et al., 1997). This marked difference corroborates the findings described in several other mycoplasma strains (Fraser et al., 1995; Himmelreich et al., 1996; Glass et al., 2000; Minion et al., 2004). The single $\sigma$ factor found in mycoplasmas is homologous to the $\sigma^{70}$ type factor found in $E$. coli or the $\sigma^{\mathrm{A}}$ vegetative type of gram-positive bacteria, and preliminary evidence for the presence of a sigma factor inducing mobility has been reported in M. pneumoniae (Bornberg-Bauer and Weiner III, 2002). Modulation of promoter selectivity of RNA polymerase by replacement of the sigma subunits is an efficient way of altering the global pattern of gene expression in response to changes in environmental conditions. Thus, the presence of only one sigma factor in mycoplasmas suggests that the level of expression of alternative sigma factors does not control either the response to external stimuli in these organisms (Razin et al., 1998) or promoter selectivity of polymerases with alternative $\sigma$ fac-

Table 1 - ORFs related to transcription in M. hyopneumoniae J and 7448 and M. synoviae strains, based on COG (NCBI) assignment of sequences.

\begin{tabular}{|c|c|c|c|c|c|c|}
\hline \multirow[b]{2}{*}{ Function } & \multicolumn{2}{|c|}{ M. hyopeumoniae $\mathrm{J}$} & \multicolumn{2}{|c|}{ M. hyopneumoniae 7448} & \multicolumn{2}{|c|}{ M. synoviae } \\
\hline & Gene & ORFs & Gene & ORFs & Gene & ORFs \\
\hline DNA-directed RNA polymerase alpha subunit & rpoA & MHJ0164 (1) & rpoA & MHP0168 & rpoA & MS0574 \\
\hline DNA-directed RNA polymerase beta subunit & rpoB & MHJ0618 (1) & rpoB & MHP0617 & rpoB & MS0485 \\
\hline DNA-directed RNA polymerase beta' subunit & rpoC & MHJ0617 (1) & rpoC & MHJ0616 & rpoC & MS0484 \\
\hline DNA-directed RNA polymerase sigma factor & rpo D & MHJ0056 (1) & rpo D & MHP0060 & $r p o \mathrm{D}$ & MS0455 \\
\hline Exoribonuclease $\mathrm{R}$ & & MHJ0033 (2) & & MHP0037 & & MS0301 \\
\hline Heat-inducible transcription repressor & $h r c \mathrm{~A}$ & MHJ0010 (2) & $h r c \mathrm{~A}$ & MHP0010 & $h r c \mathrm{~A}$ & MS0353 \\
\hline Ribonuclease III & & MHJ0411 (1) & & MHP0398 & & MS0675 \\
\hline Serine/threonine protein kinase & & & & & & MS0121 (1) \\
\hline Transcriptional accessory protein & & & & & & MS0548 (2) \\
\hline Transcription elongation factor & $\begin{array}{l}\text { greA } \\
\text { nus A }\end{array}$ & $\begin{array}{l}\text { MHJ0667 (1) } \\
\text { MHJ0586 (1) }\end{array}$ & $\begin{array}{l}\text { greA } \\
\text { nus A }\end{array}$ & $\begin{array}{l}\text { MHP0668 } \\
\text { MHP0586 }\end{array}$ & $\begin{array}{l}\text { greA } \\
\text { nus A }\end{array}$ & $\begin{array}{l}\text { MS0379 } \\
\text { MS0685 }\end{array}$ \\
\hline Transcription antitermination protein & nusG & MHJ0637 (2) & nusG & MHP0637 & nus G & MS0361 \\
\hline Transcription termination factor & & MHJ0158 (1) & & $\begin{array}{c}\text { MHP0162 } \\
\text { MHP0585 (1) }\end{array}$ & & MS0324 \\
\hline Putative transcriptional regulator & & $\begin{array}{l}\text { MHJ0271 (2) } \\
\text { MHJ0555 (2) } \\
\text { MHJ0515 (1) } \\
\text { MHJ0634 (2) } \\
\text { MHJ0303 (2) }\end{array}$ & & $\begin{array}{l}\text { MHP0279 } \\
\text { MHP0551 } \\
\text { MHP0517 } \\
\text { MHP0633 } \\
\text { MHP0311 }\end{array}$ & & $\begin{array}{l}\text { MS0024 } \\
\text { MS0024 } \\
\text { MS0195 } \\
\text { MS0095 }\end{array}$ \\
\hline & & & & & & $\begin{array}{l}\text { MS0006 (2) } \\
\text { MS0109 (2) } \\
\text { MS0127 (2) } \\
\text { MS0548 (2) }\end{array}$ \\
\hline
\end{tabular}

Numbers in parentheses represent (1) proteins whose function was determined experimentally in or purified from other bacteria; (2) proteins whose function was deduced from sequence comparison using NCBI's BLASTp. 
tors. However, the genome sequences of $M$. pneumoniae, $M$. genitalium, $M$. gallisepticum, and $U$. urealyticum contain a gene homologous to the $\delta$ subunit or $\sigma^{\mathrm{E}}$ (rpoE), as deduced from their gene sequences, that is required for the expression of several stress-response genes in E. coli and some other bacteria (Muto and Ushida, 2002).

The genomic sequences of both $M$. hyopneumoniae and $M$. synoviae lack several major regulators of gene expression, including two-component regulatory systems and multiple $\sigma$ factors. Gene promoters of $M$. hyopneumoniae and $M$. synoviae contain two distinct sequence motifs that reside, respectively, -10 and -35 regions upstream of the transcriptional start site. Primer extension studies conducted by Weiner III et al. (2000) have shown that, although a strong consensus - 10 region (TATA/TATTTTTT AAAATTAA) could be seen in M. pneumoniae, there was only a weak consensus in the -35 region (CATAATTTTA ATTTTTGAAATA/TTTT). It was also observed that there are a high proportion of transcripts with heterogeneous 5 , ends derived from initiation of transcription at reduced levels between 1 and 4 bases 5 ' to the major starting point. In addition to this apparently unique feature, in $M$. pneumoniae a high proportion of transcripts lacked an untranslated 5' leader region that could contain a ribosomal binding site (Weiner III et al., 2000). Such leaderless transcripts are rarely seen in other bacterial species and seem to be an accurate regulatory mechanism of gene transcription in Mycoplasma, although there is no confirmation of this role so far. The unusual $\mathrm{A}+\mathrm{T}$ content of intergenic regions in mycoplasma (Muto and Osawa, 1987) and the weak -35 consensus (Weiner III et al., 2000) prevents an efficient prediction of promoters by current bioinformatics tools. Moreover, the likely occurrence of cryptic operons with dysfunctional promoters resulting from mutations in the regulatory regions complicates matters further. In vivo analysis of promoter activity in mycoplasmas is presently difficult; as a plasmid transformation system is not in place, a more expedite understanding of this fundamental aspect of transcription initiation is not possible.

It is well known that there are two different mechanisms of transcription termination in E. coli, one rhodependent and one rho-independent (Opperman and Richardson, 1994). Such regulatory events are characterized by the presence of a hairpin loop in the secondary structure of mRNA, in rho-independent termination, while in the rhofactor-dependent process transcription is terminated by the interaction of the rho-factor protein with RNA polymerase. These general mechanisms are used for transcriptional regulation in a wide range of prokaryotes, although no mollicute genome sequenced so far seems to possess the rho protein. Transcription termination modulators NusA and factor $\mathrm{G}$ (greA gene), elongation factors, and NusG, which is involved in antitermination, are proteins involved in transcription in M. hyopneumoniae strains $\mathrm{J}$ and 7448 and in M. synoviae (Table 1). Gene homologs for nusA, greA and nus $G$ are also found in nine other sequenced mollicute genomes (Fraser et al., 1995; Himmelreich et al., 1996; Glass et al., 2000; Chambaud et al., 2001; Sasaki et al., 2002; Papazisi et al., 2003; Jaffe et al., 2004; Minion et al., 2004; Westberg et al., 2004), with the exception of nus $G$ in $M$. genitalium. In contrast, nusB, a transcription terminator that acts by modulating the efficiency of transcriptional antitermination, is present only in M. mycoides, $M$. penetrans and $U$. urealyticum/parvum. Washio et al. (1998) suggested that M. pneumoniae and M. genitalium may not rely on hairpin formation in transcription termination, based on calculations of free energy values around the stop codons over the entire genome. Nevertheless, it should be noted that the lack of apparent free energy drop does not necessarily indicate the complete absence of hairpins, since some of the species may have only small or weak hairpin formation at the transcription termination sites. In fact, in vitro analysis of transcription in the M. capricolum $\mathrm{RRNA}^{\mathrm{Trp}}$ gene cluster indicated that termination takes place at the T-stretch region soon after the dyad-symmetrical structure that leads to the hairpin loop formation (Yamao et al., 1988).

Recently, Benders et al. (2005) established a detailed transcriptional profile of the phylogenetically conserved $f t s Z$ cell division gene cluster in both $M$. genitalium and its closest relative, M. pneumoniae. Initiation and termination points were determined for the cell division cluster that comprises just four genes, with the fts $Z$ gene at the 3' end. Transcription of this cluster in both these organisms was shown to be highly strand-specific. While the four genes in this cluster are cotranscribed, their transcription unit also includes two additional genes of close proximity, yet with related function. Two amino acid permeases belonging to the same COG are separated from the ftsZ cluster by only $45 \mathrm{bp}$ (M. genitalium) or $42 \mathrm{bp}$ (M. pneumoniae). A transcription initiation point immediately upstream of these two genes was detected in M. genitalium, but not in $M$. pneumoniae. In $M$. pneumoniae, transcription of these genes that comprise the cell division cluster terminates at a poly(U)-tailed hairpin. In M. genitalium, this transcription terminates at two closely spaced points by an unknown mechanism. The authors concluded that groups of functionally related genes in M. genitalium and M. pneumoniae are often preceded by promoters, but rarely followed by terminators. This causes functionally unrelated genes to be commonly cotranscribed in these organisms. Due to the presence of few signals for discrete and efficient transcription termination, many genes may be transcribed by "run-on" transcription from upstream promoters. Real-time reverse transcription-PCR analysis of the $f t s Z$ gene cluster in $M$. pneumoniae showed that the mRNA levels for all six genes 
vary at most fivefold and form a gradient of decreasing quantity with the increasing distance from the promoter at the beginning (Benders et al., 2005).

Although limited to one gene cluster comprising six genes, Benders et al. (2005) provided a new understanding of the coordination of gene expression in mycoplasmas. Based on the results from M. pneumoniae and $M$. genitalium, as expected, these bacteria cotranscribe genes with related functions. However, these bacteria also cotranscribe genes that are not functionally related, and it has been proposed that this may be the rule rather than the exception in these organisms. If there are few terminators in the genomes of mycoplasmas, as previously proposed (Washio et al., 1998), then the position of these elements may not be relevant to the functional grouping of genes. Regardless of whether two genes are cotranscribed, a promoter between the two may indicate that they are not functionally related. For instance, cotranscription evidence suggests that the ribosomal protein in the $h m w$ cluster is part of an operon with upstream genes. However, it has its own promoter and is the 3'-terminal gene in the cluster (Benders et al., 2005).

It has further been proposed that $M$. genitalium makes heavy use of operon systems, potentially reducing the number of regulatory elements required for controlling the transcription of genes (Peterson et al., 1993). The arrangement of ORFs is such that there are rarely more than a few nucleotides between the stop codon of one ORF and the methionine of the next. Data taken from a recently developed operon database (Alm et al., 2005) confirm the assumption of heavy use of operons in mycoplasmas. Operon prediction data for M. hyopneumoniae strains $\mathrm{J}$ and 7448 and $M$. synoviae reveal that $70 \%, 74 \%$ and $79 \%$ of their genes, respectively, are organized in operons. These numbers compare to the $74 \%$ of operon usage in M. pneumoniae and are significantly higher than in other bacteria, such as E. coli (63\%) and Bacillus subtilis (58\%). For genes to be included in the database as part of an operon, prediction of gene pairs uses statistical inference based on distance, comparative features (how often their orthologs are near each other - within $5 \mathrm{~kb}$ - in other genomes), functional similarity (whether their predicted functions are in the same COG category), and similarity of CAI (codon adaptation index), a measure of synonymous codon usage (Price et al., 2005).

Many rRNA and tRNA genes in bacteria are clustered forming operons. Like other eubacteria, the rRNA genes of most mycoplasmas are organized in an operon and in the same order, 16S-23S-5S (Muto and Ushida, 2002). However, in Mycoplasma hyopneumoniae strains J, 7448 and 232 , only genes for $16 \mathrm{~S}$ and $23 \mathrm{~S}$ are in the same operon, and the $5 \mathrm{~S}$ gene is some $100 \mathrm{~kb}$ further downstream. Detailed organization of theses genes as well as of others involved in translation in M. hyopneumoniae strains $\mathrm{J}$ and 7448 and $M$. synoviae is presented in an accompanying paper (Santos et al., 2006).

The regulatory mechanisms involved in the control of prokaryotic gene expression are limited, since the bacterial genome is associated directly with the protein synthesizing machinery. In general, every step that is required to make an active gene product can be the focus of a regulatory event, but in practice most bacterial regulation occurs at the transcriptional level. In the last decade, complete genome sequencing of several mycoplasma strains and elaborate experimental data revealed some unique features of mycoplasma gene expression regulation (Weiner III et al., 2003; Benders et al., 2005). A survey of the mycoplasma genomes sequenced to date reveals a lack of several major gene expression regulators, including two-component regulatory systems and, as seen above, multiple $\sigma$ factors. In addition, the high $\mathrm{A}+\mathrm{T}$ content of their genomes has been found to result in adventitious promotion of transcription when fragments of their genomic DNA were introduced into $E$. coli. (Weiner III et al., 2003). The apparent scarcity of regulatory genes functioning as sensors to environmental stimuli and of genes encoding transcriptional factors suggests, but does not rule out, that adaptation of mycoplasmas to the changing environment is not per se a response to signals (Rottem, 2003). In the genome of M. hyopneumoniae strain 232, no PROSITE AraC, LysR, GntR, LuxR or sigma 54 interaction domains were found (Minion et al., 2004). Also, a search for helix-turn-helix (HTH) motifs revealed numerous AraC motifs with low scores, and consequently these proteins have a low probability of having a regulatory function. On the other hand, Papazisi et al. (2003) found 21 proteins (excluding sigma 70, recombinases, helicases and other enzymes involved in nucleic acid modification) that contained HTH motifs similar to the PROSITE AraC, LysR, GntR and LuxR motifs, even though the degree of identity is not presented.

The number of putative regulatory proteins present in the two strains of $M$. hyopneumoniae and in M. synoviae is very low (Table 1), based on COGs assigned to sequences. In either strain of $M$. hyopneumoniae, only five proteins possessing DNA-binding regulatory motifs were found compared to eight found in M. synoviae. Searching for regulatory proteins in the genome of other completely sequenced mycoplasmas showed the presence of fur (ferric uptake regulator), fruR (transcriptional repressor fructose operon) and a sucrose PTS repressor in M. mycoides (Westberg et al., 2004); a transcription regulator of the MarR family in M. penetrans (Sasaki et al., 2002); and trsE (transfer complex protein) in M. pneumoniae (Dandekar et al., 2000). No homologs of such genes were found in $M$. hyopneumoniae or in M. synoviae. 
The minimal set of regulatory proteins identified in completely sequenced mycoplasma genomes, including $M$. synoviae and M. hyopneumoniae strains J and 7448 , could be either the result of overlooking by usual computational genome analysis, the result of a remarkable adaptation to constant environments, or both. The price for the drastic saving in genes in mycoplasmas can be questioned, as this reduction may render them less adaptable to environmental changes. If so, the mechanisms for maintaining cellular homeostasis in mycoplasmas are not obvious. Answering these questions and unraveling the subtleties of the interconnected regulation mechanisms in mycoplasmas is expected to be a demanding enterprise (Razin et al., 1998). Comprehensive comparative analysis of genomic sequences and the proteins they encode is an absolute prerequisite to further advances in our understanding of cell biology. It is expected that novel mechanisms of transcription initiation and/or a refinement of the current understanding of the transcriptional process in mycoplasmas will be achieved in the coming years, as more elaborate techniques for the study of gene expression in mycoplasmas are being employed. Ultimately, increased knowledge of gene expression in these minimalist organisms should help us understand how they maintain their lifestyle.

\section{Acknowledgements}

This work was supported by MCT/CNPq and SCT/ FAPERGS within the context of the Brazilian National Genome Project. J. E. Gabriel is recipient of a CNPq DTI fellowship.

\section{References}

Alm EJ, Huang KH, Price MN, Koche RP, Keller K, Dubchak IL and Arkin AP (2005) The microbes online web site for comparative genomics. Nucleic Acids Res 33:880-892.

Benders GA, Powell BC and Hutchison III CA (2005) Transcriptional analysis of the conserved ftsZ gene cluster in Mycoplasma genitalium and Mycoplasma pneumoniae. J Bacteriol 187:4542-4551.

Blattner FR, Plunkett III G, Bloch CA, Perna NT, Burland V, Riley M, Collado-Vides J, Glasner JD, Rode CK, Mayhew GF, et al. (1997) The complete genome sequence of Escherichia coli K-12. Science 277:1453-1474.

Bornberg-Bauer E and Weiner III J (2002) A putative transcription factor inducing mobility in Mycoplasma pneumoniae. Microbiol 148:3764-3765.

Chambaud I, Heilig R, Ferris S, Barbe V, Samson D, Galisson F, Moszer I, Dybvig K, Wróblewski H, Viari A, Rocha EPC and Blanchard A (2001) The complete genome sequence of the murine respiratory pathogen Mycoplasma pulmonis. Nucleic Acids Res 29:2145-2153.

Dandekar T, Huynen M, Regula JT, Ueberle B, Zimmermann CU, Andrade MA, Doerks T, Sánchez-Pulido L, Snel B, Suyama YP, et al. (2000) Re-annotating the Mycoplasma pneumoniae genome sequence: Adding value, function and reading frames. Nucleic Acids Res 28:3278-3288.
Fraser CM, Gocayne JD, White O, Adams MD, Clayton RA, Fleischmann RD, Bult CJ, Kerlavage AR, Sutton G, Kelley JM, et al. (1995) The minimal gene complement of Mycoplasma genitalium. Science 270:397-403.

Glass JI, Lefkowitz EJ, Glass JS, Heiner CR, Chen EY and Cassell GH (2000) The complete sequence of the mucosal pathogen Ureaplasma urealyticum. Nature 407:757-762.

Himmelreich R, Hilbert H, Plagens H, Pirkl E, Li BC and Herrmann R (1996) Complete sequence analysis of the genome of the bacterium Mycoplasma pneumoniae. Nucleic Acids Res 24:4420-4449.

Jaffe JD, Stange-Thomann N, Smith C, DeCaprio D, Fisher S, Butler J, Calvo S, Elkins T, FitzGerald MG, Hafez N, et al. (2004) The complete genome and proteome of Mycoplasma mobile. Genome Res 14:1447-1461.

Koonin EV (2000) How many genes can make a cell? Annu Rev Genomics Hum Genet 01:99-116.

Kunst F, Ogasawara N, Moszer I, Albertini AM, Alloni G, Azevedo V, Bertero MG, Bessieres P, Bolotin A, Borchert S, et al. (1997) The complete genome sequence of the grampositive bacterium Bacillus subtilis. Nature 390:249-256.

Minion FC, Lefkowitz EJ, Madsen ML, Cleary BJ, Swartzell SM and Mahairas GG (2004) The genome sequence of Mycoplasma hyopneumoniae strain 232, the agent of swine mycoplasmosis. J Bacteriol 186:7123-7133.

Muto A and Osawa S (1987) The guanine and cytosine content of genomic DNA and bacterial evolution. Proc Natl Acad Sci USA 84:166-169.

Muto A and Ushida C (2002) Transcription and translation. In: Muto A and Ushida C (eds) Molecular Biology and Pathogenicity of Mycoplasmas. Kluwer Academic Publishers, Norwell, pp 323-345.

Opperman T and Richardson JP (1994) Phylogenetic analysis of sequences from diverse bacteria with homology to the Escherichia coli rho gene. J Bacteriol 176:5033-5043.

Papazisi L, Gorton TS, Kutish G, Markham PF, Browning GF, Nguyen DK, Swartzell S, Madan A, Mahairas G and Geary SJ (2003) The complete genome sequence of the avian pathogen Mycoplasma gallisepticum strain. Rev Microbiol 149:2307-2316.

Peterson SN, Hu P-C, Both KF and Hutchinson III CA (1993) A survey of the Mycoplasma genitalium genome by using random sequencing. J Bacteriol 175:7918-7930.

Price MN, Huang KH, Alm EJ and Arkin AP (2005) A novel method for accurate operon predictions in all sequenced prokaryotes Nucleic Acids Res 33:880-892.

Razin S, Yogev D and Naot Y (1998) Molecular biology and pathogenicity of Mycoplasmas. Microbiol Mol Biol Rev 62:1094-1156.

Rottem S (2003) Interaction of mycoplasmas with host cells. Physiol Rev 83:417-432.

Santos MO, Castro NS, Pereira M and Soares CM (2006) Genes involved in translation of Mycoplasma hyopneumoniae and Mycoplasma synoviae. Genet Mol Biol (in press).

Sasaki Y, Ishikawa J, Yamashita A, Oshima K, Kenri T, Furuya K, Yoshino C, Atsuko H, Shiba T, Sasaki T and Hattori M (2002) The complete genomic sequence of Mycoplasma penetrans, an intracellular bacterial pathogen in humans. Nucleic Acids Res 30:5293-5300.

Vasconcelos AT, Ferreira HB, Bizarro CV, Bonatto SL, Carvalho MO, Pinto PM, Almeida DF, Almeida LG, Almeida R, 
Alves-Filho L, et al. (2005) Swine and poultry pathogens: The complete genome sequences of two strains of Mycoplasma hyopneumoniae and a strain of Mycoplasma synoviae. J Bacteriol 187:5568-5577.

Washio T, Sasayama J and Tomita M (1998) Analysis of complete genomes suggests that many prokaryotes do not rely on hairpin formation in transcription termination. Nucleic Acids Res 26:5456-5463.

Weiner III J, Herrmann R and Browning GF (2000) Transcription in Mycoplasma pneumoniae. Nucleic Acids Res 28:44884496.

Weiner III J, Zimmerman C-U, Göhlmann HWH and Herrmann R (2003) Transcription profiles of the bacterium Mycoplasma pneumoniae grown at different temperatures. Nucleic Acids Res 31:6306-6320.
Westberg J, Persson A, Holmberg A, Goesmann A, Lundeberg J, Johansson KE, Pettersson B and Uhlen M (2004) The genome sequence of Mycoplasma mycoides subsp. mycoides SC type strain PG1T, the causative agent of contagious bovine pleuropneumonia (CBPP). Genome Res 14:221-227.

Yamao F, Iwagami S, Azumi Y, Muto A and Osawa S (1988) Evolutionary dynamics of tryptophan tRNAs in Mycoplasma capricolum. Proc Natl Acad Sci USA 82:2306-2309.

\section{Internet Resources}

National Center of Biotechnology Information, http://www.ncbi. nlm.nih.gov/genomes/lproks.cgi (October 20, 2005).

Associate Editor: Arnaldo Zaha 